\title{
LETTERS
}

\section{Is it weight loss or exercise that matters in osteoarthritis?}

In the recent review article on management of osteoarthritis, Khan and colleagues recommended weight loss as one of the noninvasive treatment modalities. ${ }^{1}$ The text and references, however, primarily discuss the effectiveness of exercise. Although there is an association between obesity and osteoarthritis of the knee, it is difficult to find convincing evidence that weight loss itself leads to improved pain or function. The referenced study by Messier and colleagues, for example, showed that the diet plus exercise group had improvement in pain and several indicators of physical function, but the diet only group showed no improvement in any outcome compared with the control group, with both groups having a similar and substantial amount of weight loss $(4.6$ and $5.2 \mathrm{~kg}$, respectively). ${ }^{2} \mathrm{~A}$ more recent study by the same author ${ }^{3}$ produced similar results despite even greater weight loss for both groups, suggesting that it is exercise, not weight loss that leads to improvement in pain and function.

Khan and colleagues also refer to the documented increased in biomechanical load on the knee in obesity, reinforcing our conventional, intuitive assumption that greater force equals greater pain and, therefore, less weight and less force will result in less pain. What is missing is clinical trial evidence that this is the case.

The distinction between advising weight loss and advising exercise is important. Not only is weight loss generally ineffective, as most patients will regain the weight within a few years, ${ }^{4}$ it is associated with substantial harms. The typical weight-cycling pattern is associated with higher long-term morbidity and mortality than stable obesity. ${ }^{5}$
The psychological effects of repeated "failure" to maintain lost weight are important and well documented. ${ }^{6}$ By advising weight loss, we fuel patients' desperate attempts at weight loss by any means and add our credible voice to the weight-shaming culture of our society.

Patients expect reliable, evidencebased information from their health care professionals. If there is evidence that advising patients to lose weight will improve symptoms of osteoarthritis, it would be helpful for the authors to share this; if not, we should leave weight out of the discussion.

\section{Ilona Hale MD}

Family physician, Department of Family Practice, Faculty of Medicine, University of British Columbia, Vancouver, BC

Cite as: CMAJ 2018 October 29;190:

E1289. doi: 10.1503/cmaj.70597

\section{References}

1. Khan M, Adili A, Winmaker M, et al. Management of osteoarthritis of the knee in younger patients. CMAJ 2018;190:E72-9.

2. Messier SP, Gutekunst DJ, Davis C, et al. Weight loss reduces knee-joint loads in overweight and obese older adults with knee osteoarthritis. Arthritis Rheum 2005;52:2026-32.

3. Messier SP, Mihalko SL, Legault C, et al. Effects of intensive diet and exercise on knee joint loads, inflammation, and clinical outcomes among overweight and obese adults with knee osteoarthritis: the IDEA randomized clinical trial. JAMA 2013;310:1263-73.

4. Diabetes Prevention Program Research Group; Knowler WC, Fowler SE, Hamman RF, et al. 10-year follow-up of diabetes incidence and weight loss in the Diabetes Prevention Program Outcomes Study. Lancet 2009;374:1677-86.

5. Tylka TL, Annunziato RA, Burgard D, et al. The weight- inclusive versus weight-normative approach to health: evaluating the evidence for prioritizing well-being over weight loss. J Obes 2014;2014:983495.

6. Phelan SM, Burgess DJ, Yeazel MW, et al. Impact of weight bias and stigma on quality of care and outcomes for patients with obesity. Obes Rev 2015;16:319-26.

Competing interests: None declared. 